\title{
Esophageal Dysphagia as the Sole Symptom in Type I Chiari Malformation
}

\author{
GRACE H. ELTA, MD, CARY A. CALDWELL, MD, and TIMOTHY T. NOSTRANT, MD \\ KEY WORDS: Chiari malformations; dysphagia, Arnold-Chiari malformations; esophageal dysmotility.
}

Swallowing and bolus movement through the oropharynx and esophagus require coordinated neuromuscular activity. Neurogenic dysphagia may arise from hemispheric, brain-stem, or lower cranial nerve lesions. Aspiration and dysphagia frequently occur in these conditions (1). This dysphagia is usually oropharyngeal rather than esophageal in origin. The rare Chiari malformations result in herniation of the hindbrain and cause dysphagia due to cranial nerve or brain-stem compression. Although these malformations represent a continuum of severity of herniation, they have been subdivided into three (or four) types depending on the extent of the herniation. The more severe types II and III Chiari malformation are associated with myelomeningoceles and commonly present in children. The milder type I Chiari malformation may present in adults or children with gradual dysphagia associated with the more common neurologic symptoms. While reports of adult Chiari malformation and dysphagia are scant in the neuroscience journals $(2,3)$, they are nonexistent in the gastroenterology literature. We describe a young woman with a three-year history of progressive dysphagia, heartburn, and weight loss and nonspecific esophageal manometric abnormalities. She had progressively severe esophageal symptoms refractory to medical, surgical, and endoscopic treatment. The subsequent development of neurological symptoms led to the diagnosis of type I Chiari malformation.

\section{CASE REPORT}

A 27-year-old woman was evaluated for a three-year history of intermittent dysphagia to both solids and liquids.

Manuscript received July 16, 1995; revised manuseript received November 30, 1995; accepted December 4, 1995.

From the Division of Gastroenterology, Department of Internal Medicine, University of Michigan, Ann Arbor, Michigan.

Address for reprint requests: Dr. Grace H. Elta, University of Michigan, 3912 Taubman Center, Ann Arbor, Michigan 481090362 .
Three years ago, upper endoscopy was performed elsewhere and was reportedly normal. She was treated with antacids and an $\mathrm{H}_{2}$ antagonist, which somewhat relieved the heartburn but did not help the dysphagia. For the previous nine months, the symptoms had become severe and occurred daily. She lost 15 pounds, which she attributed to a fear of eating due to the dysphagia. She noted that both food and liquids would stick in the mid-chest area although drinking water could occasionally wash them down. She also complained of burning pain throughout her chest that radiated into her throat. One month prior to presentation, her private gastroenterologist repeated the upper endoscopy and diagnosed "esophagitis" although no erosions were identified. A through-the-scope balloon dilator of uncertain diameter was passed through her lower esophagus, and no resistance was noted. She was given a trial of omeprazole, which again improved the heartburn, although the dysphagia continued and the omeprazole was stopped. Although she could not identify significant life stressors, an anxiolytic agent was tried without benefit. Her recent evaluation elsewhere included a normal barium swallow performed without solid phases or videofluoroscopy. Laboratory values included a normal hematocrit and white blood cell count, complement studies, serum and urine protein electrophoresis, and thyroid stimulating hormone. Rheumatoid factor, anti-ds-DNA, anti-SM, RNP, SS-A, SS-B, and $\mathrm{Scl}-70$ were negative. Arterial pressure gradient and cold temperature immersion studies were normal. She was referred for a second opinion while taking antacids as needed and ranitidine $150 \mathrm{mg}$ twice a day.

Review of systems revealed numbness of her fingertips after exposure to cold, for which the diagnosis of Raynaud's phenomenon had been entertained several years earlier. She also noted nocturnal cough and choking and a one-year history of recurrent bronchitis. Chronic headaches prompted a brain CT scan two years earlier; it was normal. Prior surgeries included repair of a deviated nasal septum and a tonsillectomy. She denied any alcohol use and had quit smoking six years ago. She worked as a medical assistant in a thoracic surgeon's office. Family history revealed a grandmother with scleroderma.

Physical examination revealed a thin but otherwise wellappearing woman. There was no thyromegaly, cervical lymphadenopathy, or tracheal deviation. The lung and heart examinations were normal. Abdominal exam demonstrated no organomegaly or tenderness. Rectal examination 


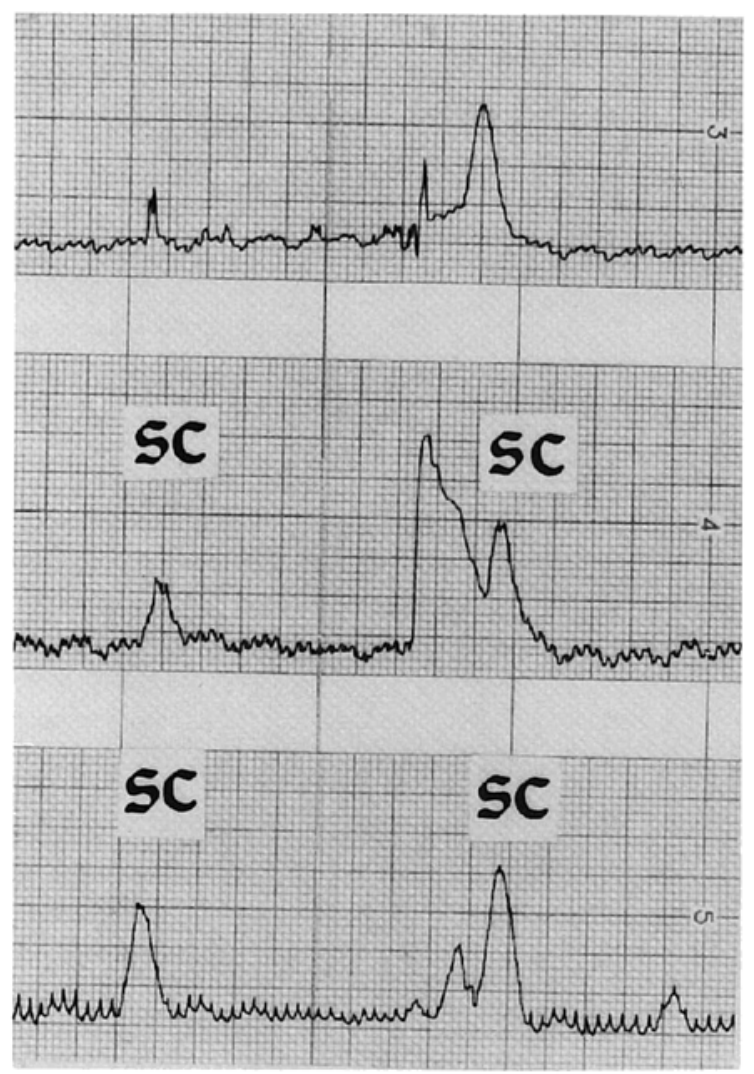

Fig 1. Preoperative esophageal manometrics showing simultaneous contractions $(\mathrm{SC})$ or nonperistaltic wave forms of the esophageal body.

showed Hemoccult-negative stool. Her extremities and skin were entirely normal. Neurological exam including cranial nerve, motor, sensory, and reflex testing was normal.

Initial manometric evaluation of the esophagus was performed with the Konigsberg solid-state probe with three transducers located circumferentially at $5-\mathrm{cm}$ intervals. Both lower esophageal sphincter (LES) and upper esophageal sphincter (UES) evaluation were done by $1-\mathrm{cm}$ sta* tion pull-throughs involving the distal two transducers. No abnormalities of the UES were detected by this technique, although UES movement would clearly bring the UES out of the examining probe. The slow station pull-through technique using both distal transducers was done to minimize movement artifact. Esophageal manometrics demonstrated normal UES pressures and relaxation with a slightly decreased LES pressure $(6-10 \mathrm{~mm} \mathrm{Hg})$. Esophageal body recordings showed simultaneous contractions with repetitive waves in the distal $15 \mathrm{~cm}$ of the esophagus. Peristalsis was maintained after only $20 \%$ of wet swallows (Figure 1 ). A 24-hr pH study showed reflux in all positions with a total time below $\mathrm{pH} 4.0$ of $11.5 \%$. The possibility of collagen vascular disease was again entertained and omeprazole 20 $\mathrm{mg}$ every day and cisapride $10 \mathrm{mg}$ four times a day was started. Due to continued chest pain and dysphagia, her doses were increased to $20 \mathrm{mg}$ twice a day omeprazole and $20 \mathrm{mg}$ four times a day cisapride two weeks later. In the subsequent month her heartburn improved but she continued to have significant dysphagia and lost an additional 5 pounds. She requested a referral to a thoracic surgeon at her employer's suggestion.

She underwent a Nissen transthoracic fundoplication without complications. Although the heartburn resolved, her dysphagia did not improve. She lost an additional 5 pounds and had bougie dilatation with 42 and 46 French Maloney dilators in the surgery clinic, but with only temporary beneficial effect. Sublingual nitroglycerin was prescribed. Thereafter the symptoms occurred with such frequency as to require weekly dilations, eventually selfadministered.

Four months after surgery and more than three years after the initial presentation with dysphagia, she had additional new symptoms. She complained of paresthesias, painful and weak extremities, head and neck aches, dizziness, and loss of balance. Progressive leg spasms and weakness occurred, leading to frequent drop attacks. Repeat collagen vascular disease work-up, thyroid studies, and an electromyelogram were normal. Neurology consultation revealed decreased vibratory sense, hyperreflexia, and a Babinski's sign. Multiple sclerosis was the initial diagnosis and a brain MRI was ordered. There was cerebellar tonsillar herniation extending $1.5 \mathrm{~cm}$ below the arch of $\mathrm{Cl}$. There was associated downward displacement of the medulla and increased angulation between the pons, medulla, and spinal cord. The findings were consistent with type I Chiari malformation. Suboccipital craniectomy was performed for brain-stem decompression with removal of the posterior arch of $\mathrm{Cl}$ and placement of a dural patch.

The patient experienced complete and immediate relief of her dysphagia that has continued for at least 12 months postoperatively. Repeat esophageal manometry two months after craniectomy used the Konigsberg probe specifically designed for UES evaluation. Typical $M$ readings were recorded and UES function and coordination were normal. Esophageal body peristalsis was now normal (Figure 2). After fundoplication LES pressures were normal (mean $20 \mathrm{~mm} \mathrm{Hg}$, peak $30 \mathrm{~mm} \mathrm{Hg}$ ).

\section{DISCUSSION}

Chiari malformations, also known as Arnold-Chiari malformations, are rare neurologic disorders due to herniation of a portion of the posterior fossa through the foramen magnum. Type I Chiari malformation is characterized by caudal descent of the cerebellar tonsils, which may be associated with a degree of medullary descent and buckling of the lower medulla. Type II Chiari malformation, which some authors further classify into types III and IV, consists of caudal displacement of the cerebellar tonsils and vermis, which are always dysplastic. The fourth ventricle descends below the foramen magnum in many instances, and in the most severe cases the pons is displaced below the foramen magnum. In contrast to 


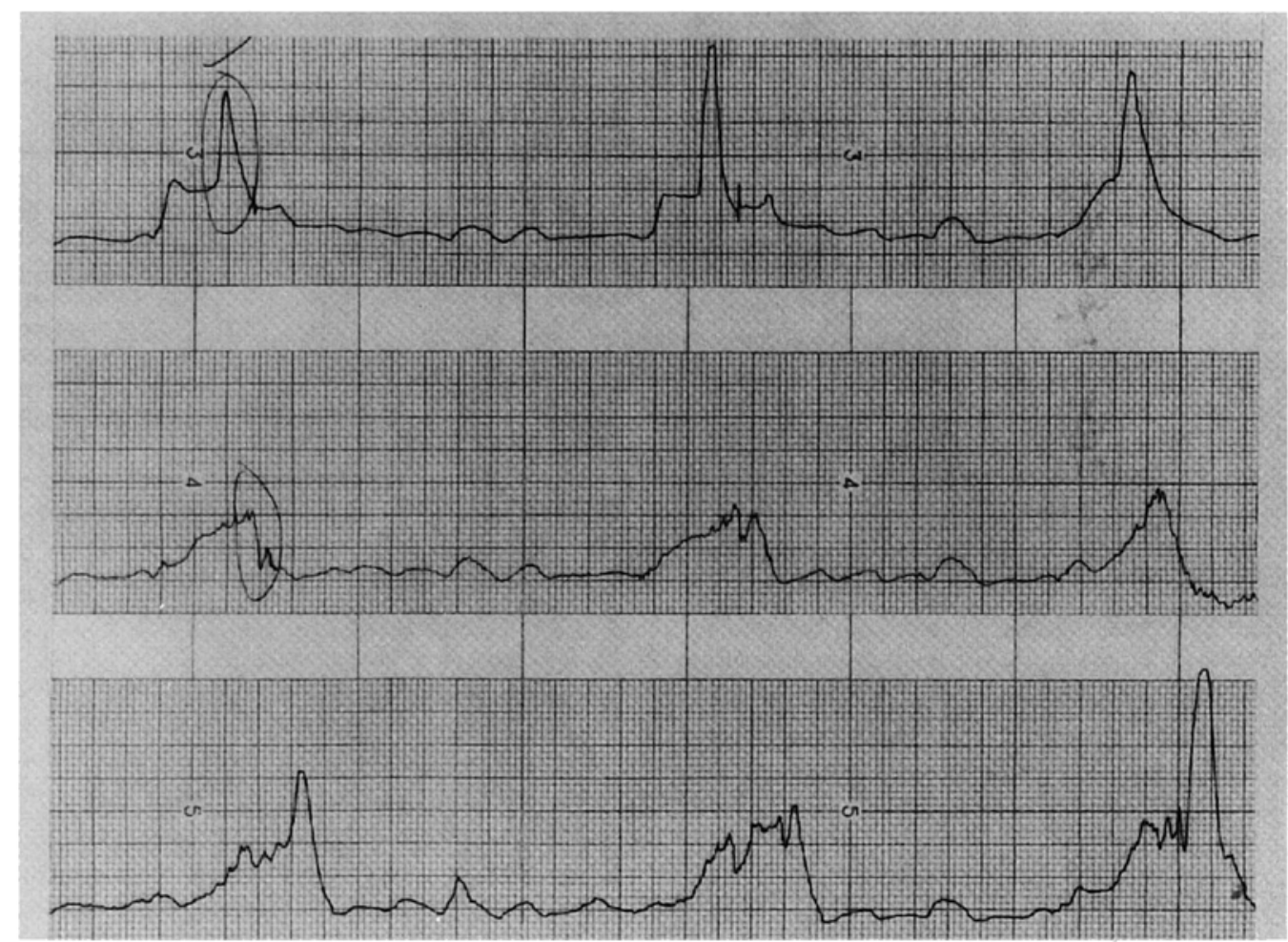

Fig 2. Esophageal manometry after suboccipital craniectomy with brain-stem decompression showing normal peristalsis with no simultaneous contractions.

the type I Chiari malformation, over $90 \%$ of the type II-IV malformations are associated with open dysraphism. These more severe types are usually diagnosed in children and present with a constellation of neurological symptoms. In contrast, the type I Chiari malformation occurs more commonly in adults.

A large review of 71 adult patients treated surgically for Chiari malformation had an age range of 15-66 years with a mean age of 43 years (2). Duration of symptoms prior to surgery ranged from $11 / 3$ months to 50 years with a mean of 9 years. The presenting symptoms include headache in $34 \%$, weakness of one or more limbs in $56 \%$, numbness of one or more limbs in $52 \%$, unsteadiness in $40 \%$, loss of temperature sensation in $40 \%$, diplopia in $13 \%$, dysphagia in $8 \%$, tinnitus in $7 \%$, vomiting in $5 \%$, and dysarthria in $4 \%$. A second review of 40 adult cases did not mention dysphagia in any of the patients (4), nor was it mentioned as a presenting symptom in a prominent neurology textbook (5). In a more recent review of 50 patients with Chiari malformation, 34 of whom were adults, three had dysphagia preoperatively although in none was this the only symptom (6).
The highest incidence of dysphagia as a presenting symptom in Chiari malformation was in a neurosurgical series where 15 of 46 patients (33\%) had symptoms of dysphagia preoperatively (3). Four of these 15 patients were adults. In eight of the 15 patients (including one adult) the swallowing impairment preceded the other brain-stem symptoms and signs.

A recent report describes two adult patients presenting with dysphagia as the sole manifestation of type I Arnold-Chiari malformation 3.5 and 5 years prior to other neurological symptoms (7). Both of these patients were diagnosed by MRI exam and had prior normal head CT exams, as did our patient. Both of these patients had improvement in their dysphagia following suboccipital craniectomy with decompression laminectomy. Another recent case report describes a 35-year-old man who presented with recurrent aspiration and a 10-year history of coughing after eating who subsequently was diagnosed with Chiari type I malformation (8). Our patient also had a history of recurrent bronchitis and coughing. It is likely that the aspiration seen in patients with Chiari malformation is mostly due to pharyngeal discoordina- 


\section{DYSPHAGIA IN CHIARI MALFORMATION}

tion, although in some patients it may be due to gastroesophageal reflux. Our patient had normal UES function on manometry with a normal barium swallow and really complained of dysphagia in the mid-chest area, suggesting that her symptoms were due to the esophageal body dysmotility and gastroesophageal reflux.

The neurogenic dysphagia that has been described in Chiari malformations includes symptoms of pooling of oral secretions, slow feeding, nasal regurgitation, and aspiration pneumonitis (3). Gastroesophageal reflux has been demonstrated in five children (three with type I and two with type II Chiari malformations) by $\mathrm{pH}$ probe or scintigraphy (9). One of these children underwent Nissen fundoplication because of aspiration. Two of these five children continued to have symptoms of gastroesophageal reflux postoperatively, raising the question of whether all of this reflux was due to the brain-stem herniation. Preoperative manometry revealed failure of relaxation of the cricopharyngeus in three of these five children. Additionally three children had esophageal body dysmotility with failure of propagation of swallows and simultaneous waves. The best described manometric abnormality in children with Chiari malformation has been cricopharyngeal achalasia (10). Indeed, cricopharyngeal myotomy has been advocated (11), although most authors reserve this surgery for patients who have spasm that is not reversed by brain-stem decompression.

The treatment of Chiari malformations is surgical decompression of the posterior fossa. Early postoperative improvement in symptoms occurs in $60-80 \%$ of patients, although relapse occurs in up to $20 \%$ (2). The best prognosis occurs in patients with less severe neurologic symptoms and signs. It has been argued that early diagnosis and surgical treatment is warranted prior to the development of irreversible neurological damage.

Dysphagia as the sole manifestation of Chiari malformations occurs rarely, although it is recognized in the neurology and neurosurgical literature. This presentation may be more common in the type I category, which is the mildest form most likely to present in adults. There are no reports in the gastroenterological literature of this entity. Severe unexplained esophageal dysmotility and dysphagia should raise the question of possible brain-stem pathology, which requires a MRI examination for diagnosis. We report a patient with severe disabling esophageal symptoms that failed to respond to both medical therapy and fundoplication in whom neurosurgical decompression resulted in complete resolution of symptoms and manometric abnormalities.

\section{SUMMARY}

Chiari malformations, also called Arnold-Chiari deformities, are rare hindbrain herniations that may present in children or adults. The most common symptoms include headache, syncope, disordered eye movement, sensory loss, weakness, and cerebellar features such as ataxia. Dysphagia occurs in 5-15\% of patients, although only a few reports describe dysphagia as the only presenting symptom. We report a case of a 27-year-old woman who presented with a three-year history of dysphagia, chest pain, and weight loss. Esophageal manometrics revealed markedly disordered esophageal motility and gastroesophageal reflux. Her symptoms failed to respond to high doses of omeprazole, prokinetics, and eventually surgical fundoplication. The subsequent onset of neurological symptoms led to the diagnosis of Chiari type I malformation. Following posterior craniotomy with decompression, her dysphagia and chest discomfort completely resolved. Repeat esophageal manometrics revealed complete resolution of prior abnormalities.

\section{REFERENCES}

1. Wiles CM: Neurogenic dysphagia. J Neurol Neurosurg Psychiatry 54:1037-1039, 1991

2. Paul KS, Lye RH, Strang FA, Dutton J: Arnold-Chiari malformation-review of 71 cases. J Neurosurg 58:183-187, 1983

3. Pollack IF, Pang D, Kocoshis S, Putnam P: Neurogenic dysphagia resulting from Chiari malformations. Neurosurgery 30:709-719, 1992

4. Eisenstat DDR, Bernstein $M$, Ross Fleming JF, Vanderlinden RG, Schutz H: Can J Neurol Sci 13:221-228, 1986

5. Williams B: Malformations. In: Swash M, Oxbury J, (eds). Clinical Neurology. London, Churchill Livingstone, 1991, pp1558-1561

6. Dyste GN, Menezes AH, VanGilder JC: Symptomatic Chiari malformations. An analysis of presentation, management, and long-term outcome. J Neurosurg 71:159-168, 1989

7. Achiron A, Kuritzky A: Dysphagia as the sole manifestation of adult type I Arnold-Chiari malformation. Neurology 10:186 187,1990

8. Nathadwarawala KM, Richards CAL, Lawrie B, Thomas GO, Wiles CM: Recurrent aspiration due to Arnold-Chiari type I malformation. BMJ 304:565-566, 1992

9. Putnam PE, Orenstein SR, Pang D, Pollack IF, Proujansky R, Kocoskis SA: Cricopharyngeal dysfunction associated with Chiari malformations. Pediatrics 89:871-876, 1992

10. Gendell HM, McCallum JE, Reigel DH: Cricopharyngeal achalasia associated with Arnold-Chiari malformation in childhood. Child's Brain 4:65-73, 1978

11. Berciano J: Dysphagia in Chiari malformations. Neurology 10:1637-1638, 1990 